\title{
Gesundheit ist ein Verb - Medizin ist mehrsprachig und vielstimmig: Chinesische und andere Perspektiven
}

\begin{abstract}
Doing Health: Chinese and Other Perspectives. In ancient China, health was related to the individual person and their unique life. Both medical and philosophical texts testify to this: Maintaining vitality in the course of one's own lifespan was a priority. Daily caring for one's health revolved around Qi 氣 a universal medium that is at the same time material and spiritual, emotional and neutral, unitary and diverse, as well as biological, psychological and physiological. Health thus becomes a verb, an act, a property to be preserved, a wavering and oscillating between pleasure and strength. Not least because of the pandemic, the demand for 'traditional' healing expertise rose worldwide. Against this background, early Chinese views on life are of unprecedented importance: From their perspective, a reorientation of public and global health policies seems inevitable.
\end{abstract}

\section{Traditionelle chinesische Vorstellungen: Das Wertvollste ist das Leben 生最貴}

Einst fragte Genghis Khan (1162-1227), ursprünglich Demüdschin, den großen daoistischen Meister Qiu Chuji (chinesisch 丘處機, 1148 -1227), auch Changchun 長春 genannt: „Vervollkommneter, welche Medizin der Unsterblichkeit (changsheng zhi yao 長生之藥, wortwörtlich „lebensverlängernde Kräuter/Langlebigkeits-Medizin“) hast Du mir von weit her mitgebracht?“ Genghis Khan hatte nämlich 1220 seinen persönlichen Minister Liu Wen beauftragt, nach Shandong zu reiten, um dort den berühmten daoistischen Meister aufzusuchen und ihn zu sich nach Samarkand zu holen. Liu Wen machte sich auf die höchst beschwerliche, mehrere tausend Kilometer weite Reise nach Shandong. Begleitet wurde er von 20 Kriegern, alle angetan mit goldenen Schildern in Gestalt von Tiger-Köpfen. Nachdem sie schließlich nach mehreren Monaten von dieser höchst gefahrvollen und kostspieligen Reise wieder in Samarkand eingetroffen waren, fand sich der daoistische Meister nun vor dem Herrscher Genghis Khan. Dessen Frage nach dem

Angelika C. Messner, Chinazentrum der Christian-Albrechts-Universität zu Kiel

Ә OpenAccess. (C) 2021 Angelika C. Messner, publiziert von De Gruyter. (cc))BY-NC-SA Dieses Werk ist lizenziert unter einer Creative Commons Namensnennung - Nicht kommerziell - Weitergabe unter gleichen Bedingungen 4.0 International Lizenz. https://doi.org/10.1515/9783110713336-011 
Elixier der Unsterblichkeit verwundert nicht, denn sein Projekt, ein eurasisches Großreich zu errichten, bedeutete, nach wirkungsvollen Strategien zu suchen, um die Macht möglichst lange zu erhalten. Das eigene Leben möglichst lange bzw. für immer zu leben, sollte hierbei helfen. Genghis Khan war in diesem Bestreben nicht allein, er konnte auf eine jahrtausendelange Tradition von Unsterblichkeitsbestrebungen chinesischer Kaiser zurückblicken. Viele unter ihnen hatten die Geheimnisse physischer Transzendenz mit Hilfe daoistischer Ratgeber zu ergründen gesucht. Nicht selten bezahlten sie für abenteuerliche Theorien und Experimente mit ihrem frühzeitigen Tod (Andreeva \& Steavu 2015). ${ }^{1}$ Genghis Khan nun erwartete eine entsprechende Antwort auf seine Frage. Der Meister aber antwortete, so haben es seine Schüler aufgezeichnet:

Ich besitze (beherrsche) den Weg des Schutzes des Lebens (weisheng zhi dao 衛生之道), aber ich besitze keine Medizin, die das Leben verlängert (chang sheng zhi yao 長生之藥) (ChihCh'ang 1931, S. 101).

Mit der Differenzierung zwischen „lebensverlängernden“ und „lebenschützenden“ Mitteln verweist der daoistische Meister auf eine Problematik, die bis heute besteht, und zwar in globaler wie in lokaler Hinsicht: die Anerkennung menschlicher Endlichkeit. Dieses endliche Leben möglichst lange zu bewahren, beruhte auf den Vorgaben von Mäßigung und Angemessenheit: Regulierung, Anpassung, Ausgleich in Essen und Trinken, Ruhen und Aktivität, und keine Exzesse (insbesondere nicht im Hinblick auf sexuelle Aktivität). Diese praktischen Leitlinien der Lebenspflege (yangsheng) finden sich implizit auch in seit über zweitausend Jahren in China belegten medizinischen Texten.

Im Blickfeld klassischer chinesischer Denk- und Orientierungsrahmen stand nicht Gesundheit, sondern das Leben. Das im heutigen Chinesischen gebräuchliche Wort für „Gesundheit“, nämlich jiankang 健康, hielt erst im frühen 20. Jahrhundert Eingang in den Sprachgebrach: Als Transfer aus der westlichen Hemisphäre (über Japan) tauchte dieser Begriff in Werbetexten für „gesunde/ hygienische Möbel bzw. gesundes Wohnambiente“ auf (Cochran 2006, S. 110). Das zweite Wort, das im modernen chinesischen Gesundheitswesen „Öffentliche

1 Es sind dies z. B. Langlebigkeitspraktiken, die insbesondere ab dem zweiten Jahrhundert nach unserer Zeitrechnung zu intensiven Experimenten mit Zinnober-Mischungen führten. Eine rotfarbige Quecksilberschwefelmischung sollte als ,Pille’ Unsterblichkeit bringen. Jedoch führte diese Pille nicht zur Unsterblichkeit, sondern häufig zum vorzeitigen Tod mehrerer Kaiser und Gelehrter der Tang-Dynastie (700 bis 900 n.u.Z.). Diese (somit) lebensverkürzenden Experimente (,wai dan 外丹“, äußeres Zinnober, äußeres Elixier) wurden ab dem 15. Jahrhundert immer weniger praktiziert. 
Gesundheitspflege“ und „Gesundheitsfürsorge“ bzw. „Hygiene/Sauberkeit“ bezeichnet, ist weisheng 衛生: Weishengju 衛生局 „Gesundheitsamt“, weishengshi 衛 生室 „Krankenstation“ und weisheng yuan 衛生院 „Krankenhaus“. Weisheng existierte, im Gegensatz zu jiankang, bereits seit vielen Jahrhunderten. Allerdings bedeutete es nicht (wie heute) Hygiene/Sauberkeit, sondern „Leben schützen“ (wei 衛sheng 生). Damit war, wie wir noch sehen werden, das Leben des einzelnen Menschen in seiner konkreten individuellen Ausprägung gemeint, und weniger die Gesundheit einer ganzen Bevölkerung.

„Was ist das Höchste/Wertvollste unter dem Himmel?“ Mit dieser Frage beginnt der fünfte von zehn Abschnitten im Buch „Zehn Fragen“ (shi wen十問) des Mawangdui-Korpus (3. bis 2. Jh. v.u.Z.). „Das Leben ist das Wertvollste“ lautet die Antwort (Zhou \& Xiao 1988, S. 379) (堯問於舜曰：「天下孰最貴？」舜曰：「生 最貴). Das Leben des Menschen, genauer das Leben eines einzelnen Menschen in seiner konkreten Form, steht hier im Fokus. Die Wertschätzung des physischen individuellen Lebens ist im religiösen Daoismus ebenso betont wie in medizinischen Wissenstexten des frühen Chinas. Einhergehend mit der Vorstellung einer vom Himmel zugewiesenen Lebensdauer enthält der Verweis auf die Wertschätzung des individuellen Lebens eine Handlungsorientierung: Es gilt, die jedem Menschen vom Himmel gegebene Lebensspanne möglichst bis zur Gänze auszufüllen (quan sheng 全生). Hierbei hilft es, sich um ein das Leben möglichst begünstigendes Ambiente zu kümmern bzw. dafür zu sorgen, dass das Lebenspotential gefördert, genährt, umsorgt, gepflegt (yang 養) wird. Die Praktiken zur Pflege des Lebens wurden erstmalig im Zhuangzi 莊子 (ca. 300 v.u.Z.) unter dem Terminus yangsheng 養生 benannt. Wichtigstes Gebot ist dabei, um die Endlichkeit des Lebens zu wissen, dann gilt es, den Geist zu nähren (yang shen 養神), und erst zum Schluss geht es darum, die Gestalt zu kultivieren (養形) (Zhuangzi 莊子 et al. 2000, juan 15) bzw. die Muskeln und Haut üppig zu machen, den Bauch zu füllen und Vorlieben und Begierden zu befriedigen.

Auch im Huangdi neijing Suwen 黄帝内经素问 (Klassiker des Gelben Kaisers, ca. 200 v.u.Z. - 200 n.u.Z. verfasst) weiß man um die Bedeutung einer korrekter Lebensführung und anderer Einflüsse auf das Lebenspotential (Cheng et al. 1982, juan 1; Unschuld 2003, S. 228). Darin wird die Tatsache, dass das Leben des Menschen endlich ist, also die Tatsache der Sterblichkeit, als conditio humana anerkannt. Gleichzeitig aber wusste man um die Möglichkeit, das Lebenspotential zu erhöhen bzw. die eigene Lebensspanne auszufüllen. In dem Buch wird berichtet, in frühen Urzeiten seien die Menschen nicht krank (bu bing 不病) geworden und in einem ihnen angemessenen Alter von etwa 100 Jahren gestorben. Aber gegenwärtig (d.h. um die Zeit 200 v.u.Z.) würden die Menschen bereits vorzeitig mit nur 50 Jahren sterben. Die Alten hätten gewusst, wie man das dao 道, den Weg des Lebens, praktiziere, und sie verstanden das Prinzip des Gleichge- 
wichts von yin 陰 und yang 陽. Sie aßen ausgewogen und regelmäßig, sie vermieden jede geistige und körperliche Überanstrengung, sie standen zu bestimmten Zeiten auf und gingen zu bestimmten Zeiten zu Bett. Sie hielten sich an die festen Rhythmen von Tag und Nacht und der Jahreszeiten: Sie schliefen zur Nacht und waren wach bei Tage und sie waren in jeder Hinsicht maßvoll. Sie bewahrten ihr Wohlbefinden. Heutzutage (also um 200 v.u.Z.) habe sich der Lebensstil der Menschen verändert: Man trinkt Wein, als wäre es Wasser, und man gibt sich zerstörerischen Aktivitäten hin. Die Menschen erschöpfen ihr jing 精 (die in den Nieren gespeicherte Essenz des Körpers) und vergeuden ihr Qi. Sie wissen nicht um die Geheimnisse der Pflege der Lebenskraft.

Sie sind nicht imstande, ihren Lebensstil und ihre Ernährung zu regulieren, und schlafen auf unangemessene Weise. Deswegen ist es nicht überraschend, dass sie mit fünfzig alt erscheinen und nicht viel später sterben (Ni 2011, S. 17).

Das Potential des Lebens zu erhöhen war grundlegend mit Techniken verknüpft, die halfen, das Qi 氣zu mehren und in fließendem Zustand zu halten. Das Qi bedeutet die alles durchflutende Kraft, Atem, Pneuma, Aktivität und Lebenskraft. Das zugehörige Piktogramm verweist auf „Abdampfen von gekochtem Reis“ oder auf „Nebel aus verdunstendem Wasser über den Reisfeldern“, und damit auf die nährende Kraft von Lebensmitteln, auf eine das Leben nährende Kraft.

Qi ist also gleichzeitig materiell und spirituell, statisch und bewegt, emotional und neutral, unitär und vielzählig, biologisch, psychisch und physiologisch, lebensfördernd, aber auch lebensbeeinträchtigend. Es ist eine Art Energie, in der alles, was geschieht, sich ereignet. Aus philologischer und etymologischer Perspektive ist es ein Universalmedium: alles ist aus Qi. Die volle Entfaltung der Lebenskraft ist nicht möglich, ohne das Qi zu schützen, es nicht zu vergeuden. In Anerkennung der eigenen Endlichkeit galt es, die Lebenszeit möglichst zur Gänze auszufüllen. Das ist die Idee, die im weitesten Sinne Gesundheit transportiert.

\section{Der Westen als Vorbild?}

In einer für das heutige China prägenden Umbruchsphase tauchte das Ideal eines Lebens ohne Krankheit wieder auf - allerdings unter ganz anderen Vorzeichen, nämlich in der Auseinandersetzung mit westlichen Denkweisen. Kang Youwei 康 有為 (1858-1927), einer der wichtigsten Reformer im China um die Wende zum 20. Jahrhundert, hatte eine Vision: eine Weltgesellschaft frei von Krankheit und körperlichen Schwächen. Bestens in westlicher Medizin ausgebildete Ärzte sollten dafür sorgen. Diese sollten außerdem dabei behilflich sein, die auf eine Insel 


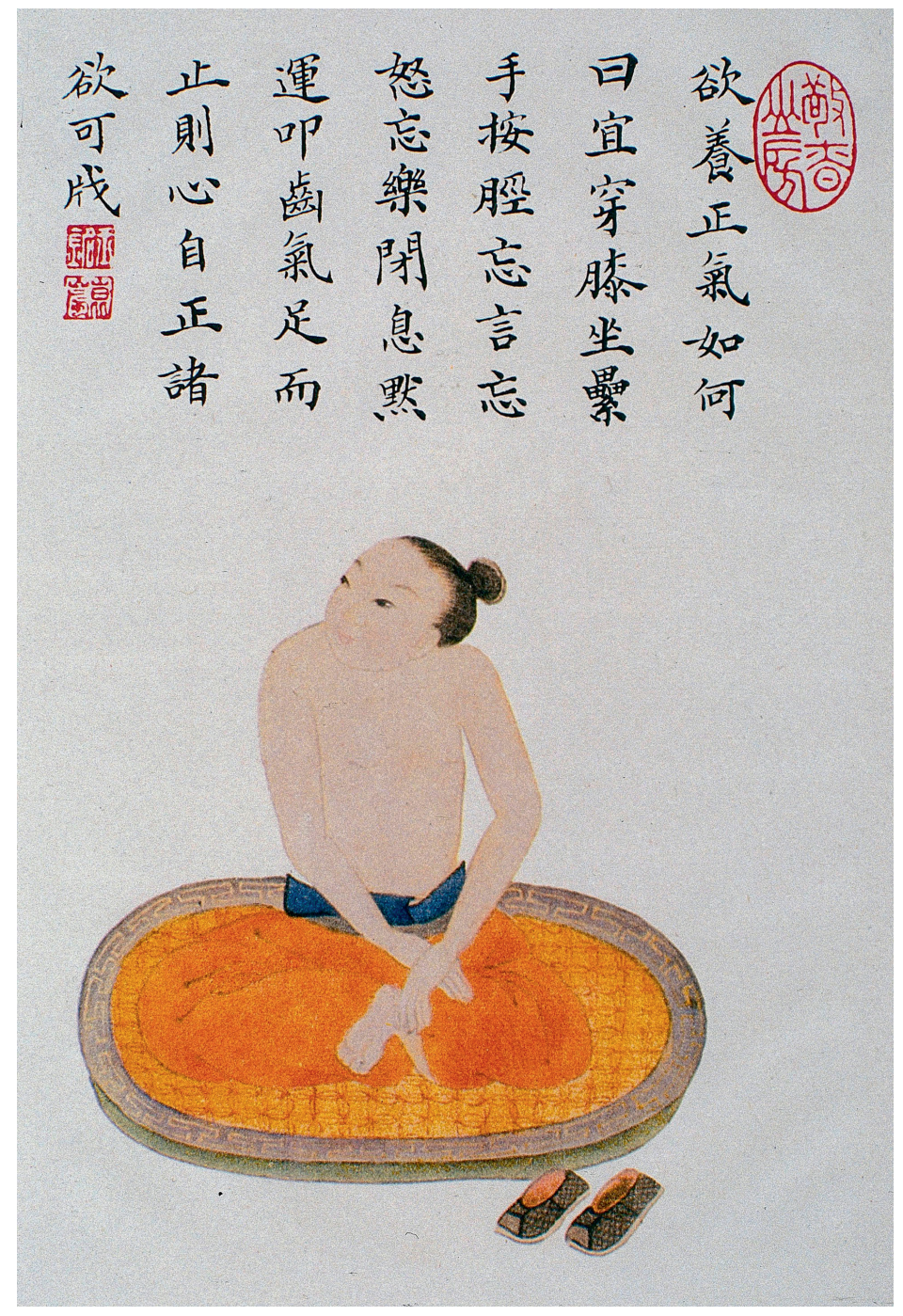

Abb. 1: Illustration aus dem Daoyin tu, einem von Kun Lan im Jahr 1875 verfasstem Buch mit 24 Techniken und therapeutischen Bewegungen zur Pflege des Qi. Die hier erklärten DaoyinTechniken umfassen 9 Sitztechniken, 6 Stehtechniken, 6 Liegetechniken, 2 Hocktechniken und eine Knietechnik. Der Text zu der hier abgebildeten Sitztechnik lautet: „Frage: Wie kann ich meine Lebenskraft festigen und vermehren? Antwort: Schlage beim Sitzen die Beine übereinander und ziehe die Füße unter die Knie. Verschränke die Hände und massiere die Unterschenkel. Vergiss das Sprechen, den Ärger und die Freude. Halte den Atem an, bewege das Qi in Ruhe und klappere mit den Zähnen. Beende (die Übung), wenn die Lebenskraft vermehrt ist, dann wird das Herz aufgerichtet sein, und alle Begierden sind entschwunden“ (übersetzt von A. Messner). Quelle: Wellcome Collection. Attribution 4.0 International (CC BY 4.0) 
deportierten Schwachen und Behinderten zu sterilisieren, um so die Vererbung von Schwäche bzw. Krankheit zu unterbinden (K’Ang 1974, S. 206/208). Die Anleihen an die im Entstehen begriffenen biologistischen, evolutionistischen und rassistischen Diskursfelder im Rahmen der Herausbildung von Nationalstaaten liegen hier klar auf der Hand. Kang Youwei hatte mit eigenen Augen die sauberen Straßen in dem von den Kolonialbehörden verwalteten Hongkong gesehen, als die Pest, 1892 von Südwestchina ausgehend, 1894 Hongkong erreicht hatte. Die sauberen Straßen waren neben der Beseitigung von „Elendsvierteln“ und einer radikal durchgesetzten Isolation und Segregation von Chinesen und Europäern Bestandteil der Hygiene-Bewegung (sanitary movement), die in den 1830er Jahren in England ihren Ausgang genommen hatte und nun, inmitten der Epidemie, zum Einsatz gekommen war.

Im Rahmen dieser Hygiene-Maßnahmen sollte das bereits ohne Pest-Epidemie „rückständige“ und „schmutzige“ China in „zivilisatorische“ Gewässer geführt werden. Die Seuchenbekämpfung wurde so, im Zeichen des Wettlaufs der Nationen, im China der Jahrhundertwende zur höchsten Priorität erklärt. Auf diese Weise konnte die chinesische Regierung die Pest-Epidemie in der Mandschurei 1910/11 mit Hilfe strikter Quarantäne-Maßnahmen, wie z. B. der abrupten Schließung der Grenzen und der Unterbindung des Eisenbahnverkehrs, zurückdrängen - und zwar ohne Hilfe ausländischer Mächte (Benedict 1996; Rogaski 2010, S. 143-144).

Nichtsdestotrotz war dies die Zeit des kontinuierlichen Niedergangs des chinesischen Kaiserreichs, im Nachgang der Opiumkriege, des japanisch-chinesischen Krieges 1894/95 und der republikanischen Umwälzung 1911/12. Vor diesem Hintergrund warben aus dem Ausland zurückkehrende Intellektuelle - gewissermaßen als die „Retter in der Not“ - für Wissenschaft und Hygiene. Das Bild des rückständigen Chinesen, menschlich und moralisch weit abgeschlagen hinter den westlichen Industrienationen, sollte nun unter den Vorgaben des Homo hygienicus (Labisch 1992) dem Typus des modernen Chinesen weichen. Dieser Prozess war langwierig und schwierig. Noch in den 1920er Jahren schreibt einer der einflussreichsten Schriftsteller im China des frühen 20. Jahrhunderts, Lu Xun 魯迅 (1881-1936):

Da das Ausland aktiv ist, ist man selbst passiv, da es die Wissenschaft pflegt, gibt man sich dem Aberglauben hin, da man dort kurze Gewänder trägt, trägt man hier lange, da man dort Gewicht auf Hygiene legt, isst man hier Fliegen; erfreut man sich dort bester Gesundheit, wird man hier krank (Lu 1979, S. 6; Messner 2000, S. 247-248; 2012).

Die aufstrebende Bakteriologie und die neue Wissenschaft (kexue 科學) sollten China retten (Eglauer 2001; Messner 2012, S. 247-264). So erfolgte eine konzeptionelle Verschränkung von bakteriologischem Wissen und dem Konzept 
einer öffentlichen Gesundheitspolitik (Public Health), die in radikaler Abkehr von allem Indigenen und „Traditionellen“ die „Bekämpfung von Epidemien“ anstrebte. Dies war die Grundlage des Dekrets, wonach im Jahr 1913 die westliche Medizin in China zum alleinigen medizinischen Studienfach wurde. Auch wenn man bereits 1922 dazu überging, die chinesische Medizin wieder als reguläres Studienfach zuzulassen (Croizier 1968, S. 47; Messner 2012, S. 247-264 und S. $247 \mathrm{ff}$ ), firmiert diese seit dem frühen 20. Jahrhundert gegenüber der westlich geprägten Medizin als unwissenschaftliche Quacksalberei. „Wissenschaft“ (kexue 科學) war das Zauberwort, das - durchgängig im vergleichenden Blick auf den Westen - alle gesellschaftlichen Probleme lösen sollte. Allen voran waren es aus dem Ausland zurückkehrende Intellektuelle und Gelehrte, die alles Traditionelle als Hindernis zum Umbau Chinas hin zur Moderne anprangerten. Die Dichotomie Ost-West verwies hier wesentlich auf „Krankheit hier - Gesundheit dort“. Lu Xun spricht in dem oben zitierten Ausschnitt das Spannungsverhältnis an, das bis heute anhält: Indem Gesundheit um die Wende zum 20. Jahrhundert aus dem Horizont ihrer jeweils partikularen sozialen, kulturellen, kosmologischen und ökologischen Bezüge gelöst wurde, wurde sie zu einer weltweiten Ware. Diese Herauslösung aber erweist sich als fatal, insbesondere jetzt im 21. Jahrhundert, nämlich angesichts einer globalen Pandemie, in der die großen Unzulänglichkeiten der medizinischen Versorgung in ihren lokalen Zusammenhängen hervortreten. Globalisiert haben sich nur die Krankheiten - Gesundheit für alle gibt es nur auf dem Papier.

\section{Gesundheit als Ware - Gesundheit als Verb}

Wenn in kapitalistisch und technokratisch organisierten Gesellschaften die menschliche Endlichkeit weitgehend negiert wird und Gesundheit unter dem Vorzeichen „bestmöglicher Optimierung“ zu einer Ware geworden ist, die schwerlich kultiviert oder „gemacht“, sondern gekauft werden will, so spiegelt dies den Überhang biomedizinisch formierter Perspektiven auf das nackte Leben (bios). Es ist aber das Leben in seinen Dimensionen kreativer Lust, das gelebt und kultiviert werden will. Und es gibt eine intrinsische Begabung zum Leben (zoe), die die Grundlage und Quelle von Wirksamkeit und Resilienz ist - diese gilt es zu fördern. Denn das Leben könnte (wieder) zur Grundlage einer Haltung werden, die Würde und eine gewisse Widerständigkeit einfordert (Farquhar \& Zhang 2012, S. 11-48).

Die Platonisch inspirierte Dichotomie zwischen bios (die biophysische Beschaffenheit von Leben/Gesundheit außerhalb der menschlichen Lebenszeit) und zoe (das vitale Leben innerhalb der Zeitspanne von Geburt und Tod) bildet die 
konzeptionelle Grundlage biomedizinischen Wirkens seit dem 19. Jahrhundert: Die Biomedizin ist damit befasst, das nackte Leben (bios) zu kurieren, aber nicht das vitale Leben (zoe) zu stärken. Die Überwindung dieser Dichotomie ist damit die Basis aller Forderungen nach einem holistischen Verständnis von Gesundheit und Krankheit (Kristeva et al. 2018, S. 1-4).Als Ausweg aus der Dichotomie verweist Julia Kristeva auf die griechische Mythologie: Demnach ist die Sorge (cura) unablässig mit dem chaotischen Zeitlichen befasst, innerhalb dessen Krankheit und Heilung gleichzeitig geschehen. Sie spielt sich in den vielen performativen Handlungen des Übersetzens zwischen den unterschiedlichen ontologischen Sphären (konkretes Leiden und die Sphäre der Götter) ab. Die Sorge ist insgesamt mit dem vitalen Leben befasst, also mit dem, was im besten Fall durch Heilkunst erreicht werden kann. Zur Überwindung der Dichotomie gilt es also, die besondere Struktur der zeitlichen und ontologischen Abgrenzungen zwischen den verschiedenen Sphären auszugleichen und zu adjustieren, um dabei gewissermaßen als Nebenprodukt die Kluft zwischen bios und zoe zu überbrücken. Es ist eine ununterbrochene Über-Setzungsarbeit (Wissens- und Sphären-Übersetzung) vonnöten, die heute als Gesundheitskompetenz bzw. Gesundheitsbildung (health literacy) bezeichnet wird. Mit dieser Daueraufgabe ist das Problem aber noch nicht gelöst. Nicht nur, dass die Gesundheitskompetenz insbesondere bei den Menschen der westlichen Hemisphäre immer weiter schwindet, es verschwindet außerdem das Gefühl der Selbst-Wirksamkeit, sowie die Grundlagen der Resilienz. Dies zeigen nicht zuletzt die Zahlen an non-communicable diseases (wie Diabetes, Bluthochdruck etc.), die etwa 71\% der Todesursachen ausmachen (World Health Organization 2018). Gesundheit als Verb verstanden bedeutet, nicht die Gesundheit als modellhaften oder absoluten Status zu begreifen, und auch nicht als höchsten Wert, sondern als Resultat ständiger Abstimmungs- und Adjustierungsverhältnisse im individuellen und sozialen Leben. Damit erst wird Gesundheit zu einem integralen Teil des Wohlbefindens im vitalen Leben während der zeitlich begrenzten Lebenszeit.

Dieses Verständnis von Gesundheit trifft sich mit dem, was weisheng (Leben-schützen) in der chinesischen Wissenssemantik meint. Die Entwicklung und Kultivierung effektiver Verhaltensweisen, die das vitale konkrete Leben im Gleichgewicht mit dem kosmischen Fließgeschehen ermöglicht, verweist auf ein Tun, das möglicherweise gesund ist, sich aber nicht in quantifizierbaren Werten (Blutspiegel etc.) erschöpfend messen lässt (Adams 2016, S. 19). Ein derartiger Gesundheitsbegriff, der das Wohlsein in Übereinstimmung mit dem dao, der Gesellschaft, der Familie und dem inneren Selbst einschließt, und sich damit dezidiert nicht außerhalb der komplexen Bereiche religiöser und philosophischer Sinnfragen oder mitmenschlicher Beziehungen und Fragen der Ökonomie und des sozialen Status bewegt, ist heute mehr denn je gefragt. 
Gesundsein: ein Verb, ein Tun, eine Eigenschaft, das Schwanken im Horizont von Lust, Kraft und vielleicht auch Freude? Es ist (mit Gadamer) etwas Verborgenes, das zu tun hat mit Vitalität (Qi), die wiederum jenseits von lokalisierten Körperteilen agiert. Diese Vitalität ist einfach da, und sie erlaubt es, über die Grenzen hinaus zu denken. Ist es möglich, gesund zu sein, jenseits der Volksgesundheit? Jenseits von Daten und Werten aus dem Labor? Wohl nur, wenn man halbwegs gesund ist. Und nur innerhalb der Grenzen des Lebens: Dschingis Khan musste dies (schmerzhaft wohl) zur Kenntnis nehmen: „Nein, lebensverlängernde Maßnahmen - darum geht es nicht. Es geht um den Schutz des Lebens, ,nähren“ und pflegen (yangsheng)“.

\section{Epilog}

Dieser Essay changiert zwischen Ost und West und zwischen unterschiedlichen Zeithorizonten. Er wurde geschrieben inmitten der Corona-Pandemie, die zu einer intensiven Kommunikation mit KollegInnen aus aller Welt geführt hat. Hierzu gehören neben Ärzten in New York, London, Mailand, Berlin, Taipeh, New Delhi, Regensburg und München auch MedizinhistorikerInnen und Medical Anthropologists in Wien (Universität Wien, Department of South Asian, Tibetan and Buddhist Studies - ISTB), in Boston (Harvard University, East Asian Languages and Civilizations), in Hong Kong (The University of Hong Kong), in London (University College of London \& Imperial College London), in Chicago (University of Chicago, Department of Anthropology), Pittsburgh (University of Pittsburgh, History Department), Beijing (China Academy of Chinese Medical Sciences), Hangzhou (Zhejiang University, Institute for Social Medicine), Singapore (National University of Singapore, Chinese Department \& Nanyang Technology University, School of Humanities \& Lee Kong-Chian School of Medicine). Besonders der Austausch mit den Kollegen in Taipeh von der Academia Sinica sowie der Taiwan Association of Traditional Chinese Medical Literature and History und dem Ministry of Health and Welfare (Bureau of Traditional Chinese Medicine) war dabei lehrreich: Während Taiwan von Beginn an strenge Maßgaben zur Prävention und Stärkung der gesamten Bevölkerung einführte und bald Corona-frei wurde, kämpfte man in anderen Teilen der Welt auf unterschiedliche Weise um eine angemessene medizinische Hilfestellung. Die Verbindung von biomedizinischer Expertise mit chinesischer Medizin in Taiwan wird auch in anderen Ländern mit Blick auf Prävention, Stärkung und Nachsorge von COVID-19 erprobt. In Mailand und in übrigen Teilen Italiens arbeiten Ärzte mithilfe narrativer Ansätze (Fondazione ISTUD 2020; Humanities Watch 2020; Marini 2016) daran, die Traumata vergangener Monate zu verarbeiten. In New York arbeiten tibetische Ärzte rund um die 
Uhr mit Patienten in unterschiedlichen Krankheitsstadien. ${ }^{2}$ In all diesen Fällen gilt es, detaillierte Protokolle über die Heilprozesse anzufertigen, damit die hier gewonnenen Erfahrungswerte in nachfolgenden Studien und Zeiten helfen können. ${ }^{3}$ Tatsächlich haben traditionelle Medizinansätze wie die chinesische und tibetische Medizin eine Bedeutung erlangt, die sich weit über ihre Heimatregion oder Communities in der Diaspora erstreckt. Längst fragen auch viele Patienten aus anderen Kulturkreisen vermehrt nach der Expertise ursprünglich bloß regional zugänglicher Medizintraditionen.

Die Pandemie 2020 und die ihr vorausgehenden Ereignisse, die sich weit zurückverfolgen lassen, haben Regionale Medizintraditionen nicht nur in China und in der westlichen Hemisphäre, sondern auch in afrikanischen Kontexten in das Zentrum globaler Aufmerksamkeit gerückt. Hierbei spielt die WHO eine zentrale Rolle, die seit 2002 in regelmäßigen Abständen Traditional MedicineStrategy Papiere lanciert. Zudem enthält das neue ICD-11 ein Modul zu Traditionellen Medizinen (International Classification of Diseases 2020). Als Meilenstein für die offizielle Anerkennung der Traditionellen Medizinen gilt gemeinhin die Verleihung des Nobelpreises für Medizin an die chinesische Malaria-Forscherin Tu Youyou im Jahr 2015.Die durch diese Ehrung aufgeworfenen Diskussionen kreisten vor allem um die Frage, inwieweit Tu Youyou in ihren Laborexperimenten auf antikes medizinisches Wissen zurückgegriffen hat, und ob sie sich tatsächlich von diesem Wissen inspirieren ließ (Hanson 2015; Unschuld 2015). Diese Fragen sind Ausdruck eines herkömmlichen Denkrahmens, der sich in einer kruden binären Unterscheidung zwischen „echter (moderner, westlicher) Wissenschaft“ einerseits und „traditionellem (unwissenschaftlichem) Wissen“ andererseits erschöpft. Im Gegensatz dazu wird die alternative Option einer medizinischen Vielstimmigkeit und Mehrsprachigkeit auf vielfältige Weise entwickelt und erprobt - in der akuten Pandemie-Situation nicht nur im chinesischen Kontext, sondern in zahlreichen Ländern der Welt. Aufbauend auf den Erfahrungen mit „traditionellen“ chinesischen Diagnose- und Heilmethoden im Zuge der SARSEpidemie 2002/03 sowie bei COVID-19-Erkrankten in China, halfen chinesische Ärzteteams auch in der westlichen Hemisphäre. Ihre Expertise kam im Iran, in Italien, Pakistan, Serbien, Venezuela, Ungarn, Nigeria und Usbekistan zum Ein-

2 Am 8. Juli 2020 fand das erste IASTAM-Webinar zum Thema „The ,Pan“ of Pandemics: Why and How Asian Approaches Matter“ statt (International Association for the Study of Asian Medicines 2020).

3 IASTAM (International Association for the Study of Asian Medicines) unterstützt gegenwärtig die medizinische Dokumentation tibetischer Ärzte in New York. Die Protokolle werden nach Beendigung der Studie in ASIAN MEDICINE, Leiden Brill veröffentlicht. https://brill.com/view/ journals/asme/asme-overview.xml. 
satz. Neben Indien suchten auch afrikanische Länder, allen voran Madagaskar, nach indigenen medizinischen Mitteln gegen COVID-19. Die tibetische Medizin wurde zudem in den Hotspots in New York und in anderen westlichen Ländern angewendet. Diese weltweite Nachfrage nach traditionellen Medizinen im Zuge der Pandemie hat nur bedingt damit zu tun, dass die Biomedizin zu Anfang kein probates Mittel gegen COVID-19 vorzuweisen hatte. Vielmehr schließt sie unmittelbar an eine im Alltag gelebte Praxis an, die sich gleichzeitig in unterschiedlichen Orientierungs- und Handlungsrahmen bewegt und sich aus verschiedenen medizinischen Expertisen bedient, ohne hierin einen Widerspruch zu sehen (Tilley 2020). Tatsächlich zeigt die neueste Forschung in Wissenschafts- und Medizingeschichte, dass das Oszillieren zwischen falsch und richtig, das beständige Adjustieren im Zeichen von Wohlbefinden und Wirksamkeit viel eher der Norm entspricht, als das Beharren auf einem einzig und alleinig richtigen Erklärungs- und Orientierungsstrang (Bian 2020, S. 108; Chiang 2019). Wir wissen heute ebenfalls, dass die vermeintlich scharfe Unterscheidung zwischen der Objektivität von Wissenschaft einerseits und der Subjektivität von Kultur, insbesondere mit Blick auf die Medizin, in die falsche Richtung weist (Napier et al. 2014). Die Lancet Commission zu Culture and Health verwies 2014 explizit darauf, dass die Trennung zwischen dem Partikularen einerseits, das bislang den Geisteswissenschaften zugerechnet wurde, und dem Universellen andererseits, das konventionell den Naturwissenschaften zugeschrieben wird, sich als obsolet erwiesen hat. Es ist dringend geboten, diese künstliche Trennlinie aufzuheben. Auch mit Blick auf die menschliche Natur, den menschlichen Leib, muss die Ontologie neu gedacht werden: Als komplexes biosoziales bzw. biokulturelles Fakt ist der menschliche Leib nicht mehr, wie seit dem 19. Jahrhundert üblich, getrennt zwischen biologischen Fakten und subjektiven (psychischen, geistigen, spirituellen) Aspekten zu behandeln. Damit sind auch die Vorannahmen der Psychosomatik hinfällig geworden, fußt diese doch auf der Dichotomie zwischen soma (Leib) und psyche (Geisthövel \& Hitzer 2019).

\section{Literatur}

Adams, Vincanne (2016): Metrics: What Counts in Global Health. Durham \& London: Duke University Press.

Andreeva, Anna/Steavu, Dominic (Hrsg.) (2015): Transforming the Void: Embryological Discourse and Reproductive Imagery in East Asian Religions. Leiden \& Boston: Brill.

Benedict, Carol (1996): Bubonic Plague in Nineteenth Century China. Stanford, CA: Stanford University Press.

Bian, He (2020): Know Your Remedies. Pharmacy \& Culture in Early Modern China. Princeton: Princeton University Press. 
Cheng, Shide 程士徳/Wang, Hongtu 王洪图/Lu, Zhaolin 鲁兆麟 (Hrsg.) (1982): Huangdi neijing Suwen. Suwen zhushi huicui 黄帝内经素问. 素問注釋匯粹 (Bd. 1). Beijing: People’s Medical Publishing House 人民卫生出版社.

Chiang, Howard (2019): The Making of the Human Sciences in China. Historical and Conceptual Foundations. Leiden \& Boston: Brill.

Chih-Ch'ang, Li (1931): The Travels of an Alchemist. The Journey of the Taoist Ch'ang-Ch'un from China to the Hindukush at the Summons of Chingiz Khan. London: George Routledge and Sons.

Cochran, Sherman (2006): Chinese Medicine Men. Consumer Culture in China and Southeast Asia. Cambridge, MA: Harvard University Press.

Croizier, Ralph C. (1968): Traditional Medicine in Modern China: Science, Nationalism and the Tensions of Cultural Change. Cambridge, MA: Harvard University Press.

Eglauer, Martina (2001): Wissenschaft als Chance: Das Wissenschaftsverständnis des chinesischen Philosophen Hu Shi (1891-1962) unter dem Einfluss von John Dewey's (1859-1952) Pragmatismus. München: Franz Steiner.

Farquhar, Judith/Zhang, Qicheng (2012): Ten Thousand Things: Nurturing Life in Contemporary Beijing. Brooklyn, NY: Zone Books.

Fondazione ISTUD (2020): „Chronicle of Healthcare and Narrative Medicine“. https://www. medicinanarrativa.eu/chronicle-of-narrative-medicine, besucht am 21.7.2020.

Geisthövel, Alexa/Hitzer, Bettina (Hrsg.) (2019): Auf der Suche nach einer anderen Medizin. Psychosomatik im 20. Jahrhundert. Frankfurt am Main: Suhrkamp.

Hanson, Marta E. (2015): „Is the 2015 Nobel Prize a Turning Point for Traditional Chinese Medicine?“. https://theconversation.com/is-the-2015-nobel-prize-a-turning-point-fortraditional-chinese-medicine-48643, besucht am 12.6.2020.

Humanities Watch (2020): „Search results for: narrative medicine“. https://humanitieswatch. org/?s=narrative+medicine, besucht am 21.7.2020.

International Association for the Study of Asian Medicines (2020): „The ,Pan“ of Pandemics: Why and How Asian Approaches Matter“. https://www.chinazentrum.uni-kiel.de/de/ aktuelles/datei-aktuelles/2020/iastam-webinar-invitation-long-version, besucht am 21.7.2020.

International Classification of Diseases (2020): „26 Supplementary Chapter Traditional Medicine Conditions - Module I“. https://icd.who.int/browse11/l-m/en\#/http\%3a\%2f\% 2fid.who.int\%2ficd\%2fentity\%2f718687701, besucht am 21.6.2020.

K’Ang, Yu-wei (1974): Ta t’ung shu: Das Buch von der Großen Gemeinschaft. Düsseldorf: E. Diederichs.

Kristeva, Julia/Moro, Marie Rose/Ødemark, John et al. (2018): „Cultural Crossings of Care: An Appeal to the Medical Humanities“. In: Medical Humanities 44(1), S. 55-58.

Labisch, Alfons (1992): Homo Hygienicus. Gesundheit und Medizin in der Neuzeit. Frankfurt am Main \& New York: Campus.

Lu, Xun (1979): Die Methode, wilde Tiere abzurichten: Erzählungen Essays, Gedichte. Berlin: Oberbaumverlag.

Marini, Maria Giulia (2016): Narrative Medicine. Bridging the Gap between Evidence-Based Care and Medical Humanities. Basel: Springer International Publishing.

Messner, Angelika C. (2000): Medizinische Diskurse zu Irresein in China (1600-1930). Stuttgart: Franz Steiner. 
Messner, Angelika C. (2012): „Contested ,Science“ Discourses on Men in 19th through Early 20th Century China“. In: Klaus Hock/Gesa Mackenthun (Hrsg.): Progress for Entangled Knowledges. Scientific Discourses and Cultural Difference. Münster: Waxmann, S. 247-264.

Napier, A. David/Ancarno, Clyde/Butler, Beverley et al. (2014): „Culture and Health“. In: The Lancet 384(9954), S. 1607-1639.

Ni, Maoshing (Hrsg.) (2011): Der Gelbe Kaiser: Das Grundlagenwerk der Traditionellen Chinesischen Medizin. München: Knaur MensSana.

Rogaski, Ruth (2010): „The Multiple Meanings of Weisheng in Manchuria“. In: Angela Ki Che Leung/Charlotte Furth (Hrsg.): Health and Hygiene in Chinese East Asia. Policies and Publics in the Long Twentieth Century. Durham \& London: Duke University Press, S. $132-159$.

Tilley, Helen (2020): „How to Make Sense of ,Traditional (Chinese) Medicine“ in a Time of Covid-19: Cold War Origin Stories and the WHO's Role in Making Space for Polyglot Therapeutics“. http://somatosphere.net/2020/tcm-covid-19.html/, besucht am 16.6.2020.

Unschuld, Paul U. (2003): Huang Di nei jing su wen. Nature, Knowledge, Imagery in an Ancient Chinese Medical Text. With an Appendix, The Doctrine of the Five Periods and Six Qi in the Huang Di nei jing su wen. Berkeley, CA: University of California Press.

Unschuld, Paul U. (2015): „Tu’s Nobel Prize Not a Win for Ancient Chinese Medicine“. https:// www.caixinglobal.com/2015-12-07/tus-nobel-prize-not-a-win-for-ancient-chinesemedicine-101046137.html, besucht am 16.6.2020.

World Health Organization (2018): „Noncommunicable Diseases“. World Health Organization. https://www.who.int/news-room/fact-sheets/detail/noncommunicable-diseases, besucht am 21.6.2020.

Zhou, Yimou 周一谋/Xiao, Zuotao 萧佐桃 (1988): Textual Research on Mawangdui Medical Book 马王堆医書考注. Tianjin: Tianjin Science and Technology Press 天津科学技术出版 社.

Zhuangzi 莊子/Lau, D.C. 劉殿爵/Wah, Ho Che et al. (Hrsg.) (2000): A Concordance to the Zhuangzi 莊子逐字索引 (Bd. 43). Hong Kong: Commercial Press. 